\title{
Abuelos, padres, hijos y nietos: un análisis generacional de las prácticas sacramentales en Ayaviri
}

\author{
Pamela Cabala Banda
}

\section{RESUMEN}

Un análisis generacional de las prácticas religiosas católicas permite conocer mejor los diversos enfoques pastorales aplicados en las últimas cinco décadas en un importante poblado del sur andino: Ayaviri, capital de la provincia de Melgar, región Puno. A partir de un significativo número de encuestas obtenidas por muestreo estadístico y entrevistas recogidas a los pobladores de la zona, este artículo ofrece información actualizada sobre las prácticas sacramentales -específicamente del bautizo, primera comunión, 
confirmación y matrimonio- de la religión andina en la zona.

Palabras clave: Sacramentos, acción pastoral, bautizo, primera comunión, matrimonio, Ayaviri.

\begin{abstract}
This generational analysis of Catholic religious practices in an important Andean town, Ayaviri (capital of Melgar, Puno) better informs us of the pastoral postures applied during the last five decades in the Andean South. Based on a significant number of surveys (which were obtained using statistical sampling methods) and interviews with the inhabitants, this paper presents valuable information about sacramental practices - especially baptism, first communion, confirmation and marriage - in the present-day Andean religion of this locale.
\end{abstract}

Keywords: Sacraments, pastoral action, baptism, first communion, marriage, Ayaviri.

\title{
INTRODUCCIÓN
}

Situado en el departamento altoandino de Puno, Ayaviri aún mantiene muchas tradiciones inveteradas, desde sus actividades de subsistencia económica — basadas en la crianza de ganado vacuno y ovino - hasta sus ritos religiosos, especialmente las festividades dedicadas cada 8 de setiembre a la Virgen de Altagracia, que se prolongan por varios días. Capital de la provincia de Melgar, este importante pueblo está ubicado en la meseta del 
Collao, a 3925 metros de altitud. Con una temperatura promedio anual de 7 grados Celsius, Ayaviri es considerado como un poblado con el ecosistema propio de la puna, piso ecológico con grandes desafíos de subsistencia y étnicamente poblado por una mayoría indígena quechuahablante. Desde el punto de vista religioso, sus habitantes profesan la religión católica. Según el XI Censo de Población y vi de Vivienda, realizado por el Instituto Nacional de Estadística e Informática del Perú (INEI) en el 2007, en la región Puno los católicos constituyen el 81,8\%, y en la zona de Ayaviri las cifras ascienden a 90\%.

Al formar parte de la zona surandina, la localidad de Ayaviri resulta ser un interesante espacio de análisis del sistema religioso andino (Marzal 2002; Geertz 1965). ${ }^{1}$ Allí uno puede encontrar evidencias propias del paradójico catolicismo andino, ya descrito por Manuel Marzal (1988), según el cual el hombre andino históricamente ha aceptado a la Iglesia católica como su propia comunidad religiosa, desarrollando un fuerte sentido de pertenencia a esta como parte de su propia cultura, por medio de una intensa actividad ritual sacramental, festiva, dedicada a los santos e incluso manteniendo vivos ancestrales ritos vinculados a los apus y la Pachamama, todo lo cual le permite entrar en contacto y comunicación con Dios (Marzal 1988).

1. La comprensión de la religión como sistema es tomada aquí de Clifford Geertz (1965: 206), quien la define como un «sistema de símbolos, que actúa para establecer en el hombre estados de ánimo y motivaciones, que son fuertes, penetrantes y duraderos». Marzal (2002: 27), basándose en Geertz, define a la religión como un «sistema de creencias, de ritos, de formas de organización, de normas éticas y de sentimientos, por cuyo medio los seres humanos se relacionan con lo divino y encuentran un sentido trascendente de la vida». 
El presente artículo proporciona información sobre la ritualidad del poblador ayavireño, abocándose solo al estudio de sus prácticas sacramentales, pues este elemento expresa de manera directa la ritualidad católica asumida por el poblador y, por ello mismo, ofrece la posibilidad de interconectar estos datos con los enfoques pastorales aplicados a lo largo de las últimas décadas. En efecto, teniendo en cuenta la importancia de los cambios ocurridos en la pastoral andina, desde los tiempos del Concilio Vaticano II hasta hoy, hemos querido ofrecer un estudio que permita dar evidencias del impacto de dichas aproximaciones pastorales en la vivencia religiosa de sus pobladores. Para ello, nos hemos basado en los resultados de la investigación titulada Análisis intergeneracional de la vivencia religiosa del poblador de Ayaviri, ${ }^{2}$ desarrollada desde el año 2009 y que encuestó a 357 personas pertenecientes a cuatro generaciones. Las encuestas se realizaron a partir de un muestreo estratificado, con un nivel de confianza del 95\%, que considera la presencia de cuatro grupos generacionales y que solo ha incluido a personas mayores de 14 años que viven permanentemente en la zona. Al ser un estudio que aplicó una metodología mixta de análisis (cuantitativo y cualitativo), las encuestas contienen preguntas cerradas y abiertas que permiten una comprensión más global del sistema

2. En esta investigación inédita participaron Alejandro Estenós, Fernando Valle, Pamela Cabala y Paloma Núñez. El trabajo fue desarrollado gracias al apoyo de la Dirección de Investigación de la Universidad Católica San Pablo, de Arequipa, que, en el marco del II Concurso de Fortalecimiento de la Investigación Científica, brindó el financiamiento. Posteriormente, se unieron al equipo Roberto Cabrera y Carlos Zegarra, quienes también proporcionaron valiosa información cualitativa. El trabajo de pesquisa contó, además, con el apoyo del Instituto de Pastoral Andina, y actualmente se encuentra en la etapa de elaboración del informe final. 
religioso, porque recoge información estadística relevante y el propio discurso del poblador.

\section{ACCión PASTORAL y POBLACIÓN LOCAL}

La evolución de las prácticas sacramentales andinas no puede ser entendida sin un análisis de los distintos enfoques pastorales presentes en la zona desde la creación de la prelatura de Ayaviri (1958), porque estos han influenciado de manera directa en los pobladores, configurando también la relación entre prácticas y creencias religiosas. No obstante, existen otras importantes variables que parcialmente podrían explicar los cambios generados en su vivencia religiosa. Uno de estos factores es la influencia de ciertos medios de comunicación que, por su reciente inserción en la zona (por ejemplo, Internet se usa solo desde comienzos de la primera década del 2000) y al ser usados principalmente por los más jóvenes, limitarían el análisis a un antes y después de su inserción, imposibilitando con ello la comparación de cuatro grupos generacionales. Una segunda variable es la presencia de las sectas en la zona, tema que tampoco analizaremos porque los pobladores de la zona se declaran principalmente católicos $(90 \%)$ y porque ello implicaría la reconstrucción histórica de la presencia de estos grupos, lo que requeriría un estudio particular.

Dado que el presente artículo está vinculado metodológicamente al análisis intergeneracional ya aludido, se propone aquí 
una segmentación etaria en cuatro generaciones, ${ }^{3}$ de modo congruente, aunque no idéntico, a la cronología fundamentada en el artículo de Alejandro Estenós y Manuel Ugarte que periodifica el desarrollo histórico de la acción pastoral en Ayaviri durante las últimas cinco décadas. ${ }^{4}$ Por ello, consideraremos, para los fines del presente trabajo, un esquema de cinco periodos: el tradicional-conservador (1959-1969), el indigenista-culturalista (1970-1974), el político-liberacionista (1975-1992), el políticodemocratizador (1993-2005) y el conservador posconciliar (2006 en adelante). ${ }^{5}$

3. La clasificación etaria propuesta en la investigación se ha realizado a partir de un trabajo exploratorio previo realizado en la zona. Además, se sustenta en las clasificaciones que el INEI desarrolla en todos sus estudios, en los que agrupa a la población mayor de 14 años en estos mismos grupos poblacionales.

4. Para ampliar el tema, ver el artículo de Estenós-Ugarte en el presente número de la revista Allpanchis, realizado a partir de su estudio La Iglesia católica en el altiplano: cinco décadas de trabajo pastoral en la prelatura de Ayaviri (19592009), llevado a cabo en el 2009.

5. Al ser un estudio descriptivo, la investigación desarrollada por el equipo de investigación de la Universidad Católica San Pablo presenta estos tipos de acción pastoral, pero no realiza una vinculación directa con la variable generacional como la presentamos en el presente artículo. La relación causal entre ambas variables que aquí se propone se ha realizado porque consideramos que existe una vinculación directa entre ambas, y ello se expresa en las estadísticas que se presentarán a lo largo de todo el artículo. 


\section{Cuadro 1}

ACCIÓN PASTORAL SEGÚN PERÍODO DE ACTUACIÓN

Y GENERACIÓN EN LA QUE INFLUYE

\begin{tabular}{|c|c|c|}
\hline Tipo de acción pastoral & Período & Generación en la que influye \\
\hline Tradicional-conservadora & 1959 a 1969 & 1. a generación (60 o más años) \\
\hline Indigenista-culturalista & 1970 a 1974 & 2. a generación (45 a 59 años) \\
\hline Político-liberacionista & 1975 a 1992 & \\
\hline Político-democratizadora & 1993 a 2005 & \\
\hline Conservadora posconciliar* & 2006 a la fecha & 4. ${ }^{a}$ generación (15 a 24 años) \\
\hline
\end{tabular}

* El regreso al enfoque conservador constituye el quinto tipo de acción pastoral, que no ha sido desarrollado en el trabajo de Estenós y Ugarte (2009), pero que hemos agregado al presente artículo porque la pastoral actual del obispo Kay Schmalhausen está desligada de todo tipo de discurso de reivindicación político-social, promoviéndose más bien una mirada «hacia dentro», hacia las normas y preceptos de la Iglesia tradicionalmente ortodoxos. Por ello, consideramos que es un regreso a la ortodoxia inicial.

Fuentes: Estenós-Ugarte (2009).

Investigación Análisis intergeneracional de la vivencia religiosa del poblador de Ayaviri.

Elaboración propia.

Como se desprende del cuadro, las personas que conforman el primer grupo generacional (60 años o más) recibieron influencia de una pastoral tradicional-conservadora, pues, iniciado el período, estaban en plena juventud, con una edad entre 15 y 24 años. El segundo grupo generacional son personas que tienen actualmente entre 46 y 59 años, y recibieron de jóvenes la influencia de una pastoral condicionada por una perspectiva indigenista-culturalista. Dado que la primera comunión representa, probablemente, el primer momento fuerte de adoctrinamiento religioso, se ha considerado en esta etapa a los nacidos en los 
últimos años de este período — por ejemplo, en 1974—, puesto que tendrían 9 años en el momento de recibir dicho sacramento. En esta fase, la administración de los sacramentos queda desligada de los colegios y se prohíbe la celebración de misas en actividades escolares. Estenós y Ugarte (2009: 36) encuentran, en su investigación, el decreto de supresión de la misa emitido por Mons. Dalle en Ayaviri, el 21 de mayo de 1973, que resuelve lo siguiente:

A partir de la fecha, no habrá celebración de Misas, en los diferentes planteles de educación, como parte de los programas de inauguración del año escolar, de clausuras, de aniversarios, del día del colegio, etc. En estas oportunidades, es muy loable que los educadores y educandos católicos, participen voluntariamente, a título personal o en grupo, a una de las misas que se celebran diariamente en la Catedral.

De este modo, la religión empezaba a ser considerada como un elemento que forma parte del espacio de decisión personal; por ello, dicho decreto concluye diciendo: «por estas medidas, queremos salvaguardar el respeto que se debe al sacrificio de la Misa y la plena libertad de conciencia que debe existir en nuestra comunidad». La tercera generación comprende a aquellas personas que tienen entre 25 y 45 años. Reciben influencia de dos acciones pastorales progresistas, que son denominadas por Estenós y Ugarte político-liberacionistas, por su vinculación ideológica con la Teología de la Liberación, y político-democratizadora. Ambos enfoques se caracterizan por la inserción de nuevos discursos sobre la opción preferencial por los pobres — que implicaba una toma de posición eclesial en la bipolaridad de la relación 
poderoso-oprimido-, asumiendo una concepción politizada de la fe, según la cual el aspecto político precede e incluye, de algún modo, al religioso. Dentro de esta tercera generación encontramos, en primer lugar, a aquellas personas que, en su juventud, vivieron el período de la pastoral político-liberacionista y que actualmente tendrían entre 44 y 45 años. También aquí se ha seguido el criterio de incluir a los nacidos en 1975 y que tenían 9 años en 1984. En esta fase, los agentes pastorales promueven el conocimiento de los derechos del hombre, con un énfasis en el pobre, desde temprana edad, en muchas instituciones educativas. En segundo lugar, se encuentran los pobladores que en su juventud recibieron influencia del período político democratizador (1993-2005), con gran influencia de la Teología de la Liberación en los planes pastorales. ${ }^{6}$

La última generación, conformada por jóvenes de 15 a 24 años, recibe la influencia de una acción pastoral preocupada por volver a la ortodoxia tradicional, pero asumiendo los cambios del Concilio Vaticano II. El enfoque conservador es similar al de la primera acción pastoral.

La segmentación por fases pastorales también se aplica, evidentemente, a los clérigos y agentes pastorales. Se ha tomado, para este trabajo, información proveniente de los Anuarios Pontificios de la prelatura de Ayaviri sobre la confesionalidad y la

6. Por ejemplo, en el 2002, en la asamblea de la prelatura, el P. Francisco Fritsch reconocía la gran influencia de Gustavo Gutiérrez como «materia prima» de la lectura de la realidad económica y sociopolítica en el plan pastoral, proponiendo que sin una organización autónoma del pueblo no se logra la liberación del pueblo de Dios (Estenós-Ugarte 2009). 
presencia de la Iglesia en términos de parroquias, sacerdotes y religiosos, desde 1965 hasta el 2007 .

\section{Cuadro 2}

TIPOS DE ACCIÓN PASTORAL Y CARACTERÍSTICAS POBLACIONALES Y ECLESIALES

\begin{tabular}{|c|c|c|c|c|c|c|c|}
\hline \multirow{2}{*}{$\begin{array}{c}\text { Tipo de acción } \\
\text { pastoral }\end{array}$} & \multicolumn{2}{|c|}{ Período } & \multirow{2}{*}{$\begin{array}{c}\text { Católicos } \\
(\%)\end{array}$} & \multirow{2}{*}{$\begin{array}{l}\text { N. }{ }^{o} \text { total de } \\
\text { sacerdotes }\end{array}$} & \multirow{2}{*}{$\begin{array}{c}\text { N. }{ }^{\circ} \text { de } \\
\text { parroquias }\end{array}$} & \multirow{2}{*}{$\begin{array}{c}\text { Religiosos } \\
\text { varones }\end{array}$} & \multirow{2}{*}{$\begin{array}{c}\text { Religiosas } \\
\text { mujeres }\end{array}$} \\
\hline & Duración & $\begin{array}{c}\text { Año de } \\
\text { estadística }\end{array}$ & & & & & \\
\hline $\begin{array}{l}\text { Tradicional- } \\
\text { conservadora }\end{array}$ & $1959-1966$ & 1966 & 98,80 & 20 & 15 & - & 7 \\
\hline $\begin{array}{l}\text { Indigenista- } \\
\text { culturalista }\end{array}$ & $1970-1974$ & 1970 & 95,00 & 22 & 31 & 11 & 14 \\
\hline \multirow{3}{*}{$\begin{array}{l}\text { Político- } \\
\text { liberacionista }\end{array}$} & \multirow{3}{*}{$1975-1992$} & 1976 & 93,30 & 17 & 31 & 9 & 10 \\
\hline & & 1980 & 93,30 & 22 & 31 & 10 & 10 \\
\hline & & 1990 & 83,40 & 22 & 31 & 9 & 35 \\
\hline \multirow{6}{*}{$\begin{array}{l}\text { Político- } \\
\text { democratizadora }\end{array}$} & \multirow{6}{*}{$1993-2005$} & 1999 & 80,00 & 11 & 37 & 7 & 33 \\
\hline & & 2000 & 79,80 & 11 & 40 & 6 & 23 \\
\hline & & 2001 & 79,80 & 13 & 42 & 6 & 29 \\
\hline & & 2002 & 89,60 & 13 & 25 & 11 & 27 \\
\hline & & 2003 & 89,60 & 14 & 35 & 8 & 24 \\
\hline & & 2004 & 98,00 & 17 & 30 & 4 & 24 \\
\hline \multirow{2}{*}{$\begin{array}{l}\text { Conservadora } \\
\text { posconciliar }\end{array}$} & \multirow{2}{*}{$\begin{array}{l}2006 \text { en } \\
\text { adelante }\end{array}$} & 2006 & 96,00 & 17 & 32 & 6 & 21 \\
\hline & & 2007 & 96,00 & 20 & 32 & 6 & 30 \\
\hline
\end{tabular}

Fuentes: Estenós-Ugarte (2009).

Investigación Análisis intergeneracional de la vivencia religiosa del poblador de Ayaviri.

Prelatura de Ayaviri <www.prelaturaayaviri.org>.

Elaboración propia.

7. Toda la información presentada ha sido extraída de los datos publicados en los Anuarios Pontificios. 
Como se observa, la cantidad de católicos ha variado según el tipo de acción pastoral desarrollado en la zona. La pastoral tradicional-conservadora, en cuyo período $98,8 \%$ se declaraban católicos, tenía como eje central «vigilar la ortodoxia de la fe y procurar conservar la pureza de la fe». ${ }^{8}$ Posteriormente, el porcentaje de católicos va decreciendo, y llega a descender en 5\% durante la década de 1970, años de influjo de las pastorales indigenista-culturalista y político-liberacionista. Sin embargo, la crisis de la masa poblacional católica en la zona se produjo en los años ochenta, período de intenso progresismo, en el que el número de católicos bajó hasta en un 10\% más, descenso que continuaría en los noventa hasta llegar a 79,8\%. En la primera década del siglo xxi, coincidentemente con el retorno de la pastoral conservadora posconciliar, el porcentaje de católicos se incrementaría nuevamente, llegando a 96\%, cifra muy similar a la reportada en el primer período.

En la década de 1990, el número de parroquias se incrementó y el número de sacerdotes disminuyó a la mitad (de 22 en 1990 a 11 en 1999), pero se triplicó el número de religiosas mujeres respecto del período anterior. Este desbalance debe de haber provocado alguna disminución en la administración de los sacramentos y un aumento de la acción pastoral de religiosos y laicos. En efecto, las religiosas tuvieron un papel fundamental en la promoción activa de la pastoral político-liberacionista, uno de cuyos objetivos principales era la «apertura al mundo». «Falta respirar más oxígeno extra-eclesial, nos estamos

8. En el folio 4, fechado el 21 de marzo de 1966 (Estenós-Ugarte 2009). 
encerrando en nosotros mismos, nos falta preocuparnos más por la organización económica y sociopolítica de nuestro pueblo que por nuestra propia organización eclesial», opinaba la hermana Elizalde en 1987 en Sandia. El aumento de parroquias podría deberse también a una política hecha explícita en la reunión de delegados del IPA (1968), en la que se decidió «elegir una parroquia en cada una de las diócesis y prelaturas, en las que se experimentaría un nuevo modelo de presencia pastoral en el campo» (Judd 1989).

En la última década, la primera del 2000, es posible percibir un progresivo incremento del número de parroquias en la zona. En el primer período tradicional-conservador se contaba con 15 parroquias, y, con la siguiente pastoral indigenista-culturalista, el número de parroquias se duplicó a 31. Esas cifras se mantienen hasta la fecha, pero en los años 2000-2001 hubo un incremento a 40/42 parroquias, respectivamente, y luego un descenso a 25 en el 2002.

\section{El bautizo: «El SeÑor nOS RECONOCE COMO HIJOS»}

El bautismo es el primer sacramento recibido por casi la totalidad (entre el 90\% y 100\%) de las personas en sus primeros meses de vida, y constituye un eje central en la vida religiosa personal y colectiva, llegando a convertirse en un elemento constitutivo de la cultura andina (Marzal 1988). Probablemente por el peso de esa tradición, los testimonios recogidos en diferentes generaciones proporcionan información básicamente muy similar sobre la percepción del bautismo: un sacramento que posibilita ser hijos de Dios, borra los pecados y permite a los fieles pertenecer la Iglesia católica. 
En la primera generación, conformada por las personas mayores de 60 años, el bautizo es comprendido como un sacramento que genera un vínculo especial con Dios: «El bautizo es estar, pues, en la gracia de Dios porque es un sacramento que debemos cumplir y solamente así nos podemos llamar cristianos»; «es un sacramento de Dios que hay que cumplir para entrar a la casa de Dios»; «estar junto a Dios, pertenecer, estar al lado de Dios». Es concebido como un paso fundamental para ser considerado cristiano. «Yo pienso como cristiana que [el bautismo] es indispensable, justamente por el bautizo uno se vuelve cristiano».

Para la segunda generación, que agrupa a personas de entre 45 y 59 años, el bautizo es también un vínculo con Dios, pero entendido en términos de entrega y reconocimiento del Padre: «Es entregarse a Dios, seguir el camino del Señor»; «es un sacramento que nos da Diosito, el Señor nos reconoce como hijos», «es ser hijo de Dios», «hijos de la Iglesia e hijos de Cristo»; y al ser nuestro Padre, debemos seguir su palabra: «para que se cumpla la religión católica» y ello nos permitirá «entrar al reino de Dios».

Algunas personas que tienen entre 25 y 45 años entienden el bautizo «como una comunión con lo sagrado, con lo espiritual que lleva cada uno», manifestando una percepción menos personal de Dios, aunque otros expresan una relación cercana con Él: «reconocerme como hija de Dios», «haber recibido a Dios y vivir con Él, compartir mi vida con Él», «ser hija de Dios, antes de bautizarme no era nada», «pertenecer a la familia católica y seguir lo que la Iglesia nos pide y también tener una vida espiritual».

Finalmente, los más jóvenes consideran el bautizo como el primer sacramento que inicia nuestra cercanía a Dios y a la 
Iglesia: «El bautizo es como un acto de acercarse a Dios desde un principio», «es el primer sacramento que yo recibí y, bueno, siento que soy parte del cuerpo de Jesús»; «el bautizo es cuando nosotros ya nos unimos [...] a la Iglesia»; lo que nos permite ser considerados como cristianos: «el bautizo es así como una marcación de ser cristiano, ¿no?, de ser católico».

Con respecto a la edad de la administración de los sacramentos, a pesar de que un grupo de personas (cerca del 12\% del total) no recuerda la edad en que recibió el bautizo, existe una notoria diferencia entre las tres primeras generaciones y la más joven.

\section{Cuadro 3}

DisTRIBUCIÓN POBLACIONAL SEGÚN GENERACIÓN Y EDAD DE REALIZACIÓN DEL

BAUTIZO

\begin{tabular}{|c|c|c|c|c|c|c|}
\hline \multirow{2}{*}{ Generación } & \multicolumn{5}{|c|}{ Edad en que se bautizaron ( $\%)$} & \multirow[b]{2}{*}{ Total } \\
\hline & $\begin{array}{l}\mathrm{Al} \text { año } \\
\mathrm{o} \text { antes }\end{array}$ & $\begin{array}{l}\text { De } 2 \text { a } \\
10 \text { años }\end{array}$ & $\begin{array}{l}\text { De } 11 \text { a } \\
15 \text { años }\end{array}$ & $\begin{array}{l}\text { De } 15 \text { a } \\
\text { más años }\end{array}$ & $\begin{array}{l}\text { No } \\
\text { recuerda }\end{array}$ & \\
\hline $\begin{array}{l}\text { 1. }{ }^{\mathrm{er}} \text { grupo generacional } \\
\text { ( } 60 \text { o más años) }\end{array}$ & 47,9 & 33,3 & & 2,1 & 16,7 & 100,0 \\
\hline $\begin{array}{l}2 .^{\circ} \text { grupo generacional } \\
\text { (46 a } 59 \text { años) }\end{array}$ & 39,3 & 35,7 & 3,6 & 3,6 & 17,9 & 100,0 \\
\hline $\begin{array}{l}3 .^{\text {er }} \text { grupo generacional } \\
\text { ( } 25 \text { a } 45 \text { años) }\end{array}$ & 35,5 & 42,1 & 10,5 & 6,6 & 5,3 & 100,0 \\
\hline $\begin{array}{l}\text { 4. }{ }^{\circ} \text { grupo generacional } \\
\text { (15 a } 24 \text { años) }\end{array}$ & 10,6 & 53,2 & 6,4 & 14,9 & 14,9 & 100,0 \\
\hline
\end{tabular}

Fuente: Investigación Análisis intergeneracional de la vivencia religiosa del poblador de Ayaviri.

Elaboración propia. 
A pesar del proceso de secularización cultural y, contrariamente a lo que este podría dejar suponer, a partir de las estadísticas presentadas, en la cuarta generación se vuelven a recibir los sacramentos en el período temprano de la vida (cerca del 15\%). Aquellos jóvenes que lo realizaron en plena juventud tienen una reflexión más profunda sobre él, al concebirlo como «algo muy importante [...] para unirnos más con Dios» y que permite la unión y reconciliación con Dios, «estar en gracia con Dios, ser parte de Dios y pa' borrar el pecado original», «entrar a su corazón de Jesús [...] y me reconcilio».

El avance en las políticas públicas de escolaridad en el Perú ha influido en el mayor nivel educativo de los hijos respecto de sus padres. Esto es una realidad palpable en Ayaviri, y se refleja en la zona de estudio. Al indagar sobre el nivel educativo, encontramos que las generaciones más jóvenes presentan mayores niveles de escolaridad respecto de las más adultas. ${ }^{9}$ En este panorama, y a pesar de que la mayoría de la población se encuentra bautizada en los primeros años de edad, encontramos que en la cuarta generación (15 a 24 años) aquellos que tienen mayores niveles educativos (superior universitario) se han bautizado entre los 2 y 15 años. Además, del total de bautizados con estudios técnicos completos, la mitad (50\%) se bautizaron con más de 15 años. En otras palabras, los mayores niveles educativos no siempre implican mayores niveles de secularización.

9. Como muestra de ello, $15 \%$ de la primera generación (60 o más años) refirieron no haber recibido instrucción alguna y 49\% tienen estudios de primaria (completa o incompleta), mientras que en la cuarta generación (15 a 24 años) 78\% tienen secundaria completa culminada o la siguen cursando. 


\section{LA PRIMERA COMUNIÓN Y LA CONFIRMACIÓN}

Con el sacramento de la primera comunión, se acrecientan las diferencias generacionales en la zona. La pastoral indigenistaculturalista de la segunda generación propone que «la primera comunión pase a ser un compromiso personal de fe». Se buscaba más independencia en la toma de decisiones de los pobladores y que la religión pasara a un ámbito personal (de decisión privada o familiar). Por ello, se suspendió la misa en los centros educativos en fechas conmemorativas (inauguración del año escolar, aniversario, clausura, etcétera) y, cuando hubo celebraciones eucarísticas en la Catedral, se evitó imponerlas como obligatorias. Acorde con ello, se establecía que «las ceremonias de primera comunión se realizarán fuera del ambiente escolar y bajo plena responsabilidad de los padres de familia y del Párroco». ${ }^{10}$ Años después, en 1991, en la reunión pastoral zonal de la zona de Carabaya, en Ajoyani, se estableció lo siguiente:

Hay poco trabajo con los niños en los barrios y campos, hay poca relación con los profesores y padres de familia, hay poca permanencia de los acompañantes; unos delegados de niños que se sientes superiores, unos niños ricos que se retiran del grupo, unos niños que defienden sus derechos, los que no admiten unos adultos que prefieren una educación vertical y dominadora. ${ }^{11}$

10. Decreto del 21 de julio de 1973, emitido por Mons. Luis Dalle.

11. Investigación Análisis intergeneracional de la vivencia religiosa del poblador de Ayaviri. 
El principal significado asignado a este sacramento por la primera generación es la comunión espiritual con el cuerpo de Cristo y hay una clara conciencia del carácter sacramental del rito: «recibir a nuestro Señor Jesucristo por primera vez». Esta unión exige mantenerse en estado de gracia: «Cuando recibes tu comunión para comulgar y, al comulgar, tú estás con Dios siempre; todos los domingos tienes que comulgar para perdonarte de cualquier pecado que hayas cometido en la semana». Por medio de la eucaristía obtenemos perdón y nos unimos a Él: «Pienso que nos perdona Nuestro Señor nuestros pecados y ya estamos con Dios»; y permite, además, «hacernos soldados de Cristo». Se trata, entonces, de una experiencia espiritual que une a Cristo mismo, a su cuerpo sacramentado y, por ello, expía los pecados.

La segunda generación también hace referencia a la unión con Dios, pero sin una clara conciencia sacramental: «sería el encuentro con Dios», «hacer la primera comunión [...] es escuchar la misa», «la primera comunión para mí, pe, es una forma de orar, una forma de irse con Dios [...]; es la primera vez que se fusionan, que se unen». A veces, los encuestados comunican una experiencia que podría ser no tanto espiritual sino más bien psicológica: «recibir el cuerpo del Señor para sentirnos tranquilos, nos da fuerza», pero en cualquier caso expiatoria: «perdona los pecados». La tercera generación tampoco tiene claridad sobre la sacramentalidad de la eucaristía, pero resalta la expiación de los pecados y la reconciliación con Dios: «fue como reconciliarme con Dios, donde cambias, ¿no?; tienes un cambio». Mencionan, a diferencia de las anteriores generaciones, la importancia de la confesión como requisito importante de este sacramento: «para confesar nuestros pecados, para limpiarnos». 
En contraste con las dos generaciones anteriores, los jóvenes de la cuarta generación explican en su mayoría y con mayor claridad el carácter sacramental de la comunión eucarística, según los cánones conservadores, concibiéndola como una experiencia fundamentalmente espiritual: «cuando nosotros hacemos la primera comunión empezamos a recibir la hostia, el cuerpo de Cristo», «la primera comunión es dar un paso más para estar cerca de Dios, para recibir a Dios cada vez que comulgamos», y ese encuentro con Dios solo se puede realizar acudiendo a la misa: «o sea, después que hice la primera comunión todos los días voy a misa, domingos».

La recepción del sacramento de la confirmación pone en evidencia las mayores diferencias entre las prácticas rituales generacionales. Como se observa en el cuadro estadístico, no todos los que hacen la primera comunión reciben después la confirmación. La brecha entre ambos sacramentos aumenta significativamente conforme pasan las generaciones. Es verdad que, en el momento de la toma de encuestas, varios jóvenes que manifestaron estar preparándose para la confirmación o tener la intención de recibir el sacramento aún no la habían hecho. Sin embargo, es innegable que las dos últimas generaciones muestran un dramático descenso, en torno al 30\%, en la realización del sacramento. Ello puede deberse a la evidente dificultad de que solo el obispo puede administrarla, hecho que tal vez demanda una mayor organización y coordinación en parroquias y colegios. Sin embargo, es posible también que, en las últimas generaciones, se haya debilitado el sentido y significado de la confirmación en la cultura religiosa actual. 
Cuadro 4

NiVEL DE REALIZACIÓN DE LA PRIMERA COMUNIÓN Y CONFIRMACIÓN (\%)

\section{Generación}

1. ${ }^{\text {er }}$ grupo generacional (60 o más años)

2. grupo generacional (45 a 59 años)

$3 .^{\text {er }}$ grupo generacional (25 a 45 años)

4. ${ }^{\circ}$ grupo generacional (15 a 24 años)
Primera comunión Confirmación

87,5

81,6

72,2

71,2

64,9

37,5

30,0

Fuente: Investigación Análisis intergeneracional de la vivencia religiosa del poblador de Ayaviri.

Elaboración propia.

A diferencia de los demás sacramentos, cuando se les pregunta a los pobladores sobre el significado de la confirmación en sus vidas, les es imposible explicarla con claridad, dificultad que está presente en todas las generaciones, pero con mayor incidencia en las tres primeras: «La confirmación... eso ¿cómo se llama?... Eso, más bien, no le podría contestar; eso es más bien cumplir ya con la religión católica»; «la confirmación, confirma, ¿algo uno que desea?», «La confirmación es... eee... catequesis... no sé». En la última generación, sin embargo, hay mayor claridad en la concepción del sacramento, aunque tampoco se expresa la concepción oficial: la infusión del Espíritu Santo. Al momento de explicar su significado, se vincula el sacramento a un mayor compromiso con Dios y la Iglesia: «confirmar la religión católica... prefieres ser cristiano», «confirmar para siempre, para no aceptar otra religión», compromiso que generaría un mayor acercamiento con Dios y el cumplimiento de su palabra: «Es un sacramento donde confirmamos nuestra fe si realmente queremos pertenecer a la Iglesia católica», «Es acercarse más 
a Dios, es ser más cumplido». Esta situación puede deberse a dos factores: el primero es la edad cercana en que los jóvenes han realizado dicho sacramento, que les permite tener conocimientos más claros por ser más recientes y estar más frescos en la memoria; y el segundo es la presencia de una pastoral más conservadora, que prioriza la enseñanza más profunda y clara.

En efecto, a diferencia de la última generación, los discursos pastorales de las tres primeras generaciones no tocaban el tema de los sacramentos, porque centraban su atención en el compromiso social y la acción política. En el informe de las actividades institucionales del 2005, último año de actuación de la pastoral políticodemocratizadora, se menciona que «por consenso queremos promover una formación que lleve a los jóvenes al compromiso social y político desde una experiencia de identificación cristianaeclesial y desde la propia identidad cultural».

Al respecto, Esteban Judd (1989) afirma que la acción de la Iglesia en el sur andino se ha realizado de «modo periférico» en la vida de las comunidades campesinas, y que siempre fue considerada como «una institución necesaria y útil sólo para fines administrativos o específicamente sacramentales»; por ello, desde finales de los sesenta e inicios de los setenta, la Iglesia se vuelca al campo y descuida su acción sacramental, iniciándose así el desarrollo de estudios etnográficos. En el Plan Pastoral de la Prelatura de Ayaviri, realizado en Chucuito, del 18 al 22 de abril de 1977, se plantea como una de las acciones pastorales «estudiar y hacer conocer los derechos del hombre como persona». Dicha situación se ve reflejada en las cifras presentadas en el cuadro 5 . 
Cuadro 5

EDAD DE REALIZACIÓN DE LA PRIMERA COMUNIÓN SEGÚN GENERACIÓN DE

PERTENENCIA

\begin{tabular}{|c|c|c|c|c|c|}
\hline \multirow{2}{*}{ Generación } & \multicolumn{4}{|c|}{ Edad en que se hizo la primera comunión (\%) } & \multirow[b]{2}{*}{ Total } \\
\hline & $\begin{array}{l}\text { De } 08 \text { a } \\
11 \text { años }\end{array}$ & $\begin{array}{l}\text { De } 12 \text { a } \\
14 \text { años }\end{array}$ & $\begin{array}{l}\text { De } 15 \text { a } \\
\text { más años }\end{array}$ & $\begin{array}{c}\text { No } \\
\text { recuerda }\end{array}$ & \\
\hline $\begin{array}{l}\text { 1. }^{\text {er }} \text { grupo generacional } \\
\text { ( } 60 \text { o más años) }\end{array}$ & 29,3 & 36,6 & 4,9 & 29,3 & 100,0 \\
\hline $\begin{array}{l}\text { 2. } .^{\circ} \text { grupo generacional } \\
\text { (46 a } 59 \text { años) }\end{array}$ & 29,2 & 41,7 & 16,7 & 12,5 & 100,0 \\
\hline $\begin{array}{l}3^{\text {er }} \text { grupo generacional } \\
\text { ( } 25 \text { a } 45 \text { años) }\end{array}$ & 32,8 & 36,2 & 25,9 & 5,2 & 100,0 \\
\hline $\begin{array}{l}\text { 4. } .^{\circ} \text { grupo generacional } \\
\text { (15 a } 24 \text { años) }\end{array}$ & 37,8 & 24,3 & 32,4 & 5,4 & 100,0 \\
\hline
\end{tabular}

Fuente: Investigación Análisis intergeneracional de la vivencia religiosa del poblador de Ayaviri.

Elaboración propia.

El retorno a la vivencia sacramental mostrado en el bautizo por la última generación también se manifiesta no solo en la primera comunión sino también en la confirmación, aunque en cantidades aún bajas, pues 32,4\% de los jóvenes de 15 a 24 años han realizado su primera comunión en los últimos años con la nueva pastoral. La tercera generación empieza a presentar un incremento de la realización de este sacramento en la juventud o edad adulta. Los mayores niveles educativos no han significado mayor secularización, debido a que del total de jóvenes que realizaron la primera comunión teniendo más de 15 años, 67\% tienen estudios secundarios y 33\% estudios técnicos superiores. En el caso de la tercera generación (25 a 45 años), quienes la hicieron siendo mayores de 15 años tienen estudios secundarios 
$(70 \%)$, técnicos (13\%) y superiores $(27 \%)$. En la confirmación, la cuarta generación, conformada por personas de entre 14 y 17 años, se encuentra en edad de realizarla y aunque solo $30 \%$ la han realizado hasta el momento de la aplicación de la encuesta, no sabemos cuánto más se incrementarán dichas cifras, producto de la acción de la nueva pastoral.

Matrimonio: «Toda pareja debe Vivir EN LA GRACIA DE Dios»

En promedio, $82 \%$ de la población reconoce un conjunto de diferencias entre el matrimonio y la convivencia. El matrimonio es entendido por los pobladores en términos de formalidad, que permite una mayor duración de la relación, generada por la unión ante Dios, a partir de la bendición recibida por Él para ser felices. A diferencia de ello, la convivencia, que se presenta en la sociedad actual como una alternativa al matrimonio por ser más fácil, es caracterizada en términos de informalidad, lo que limita su duración a la simple decisión de quienes la realizan, y, por ende, no asegura una felicidad duradera ni para la pareja ni para los hijos. Presentamos a continuación un cuadro en el que se reporta el nivel de diferenciación que los pobladores de las distintas generaciones establecen entre el matrimonio y la convivencia. 
Cuadro 6

DISTRIBUCIÓN DE LA POBLACIÓN SEGÚN GENERACIÓN Y PERCEPCIÓN DE LA EXISTENCIA O AUSENCIA DE DIFERENCIAS ENTRE MATRIMONIO Y CONVIVENCIA

\begin{tabular}{|l|c|c|}
\hline \multirow{2}{*}{ Edad } & \multicolumn{2}{|c|}{$\begin{array}{c}\text { Diferencia entre matrimonio } \\
\text { y convivencia (\%) }\end{array}$} \\
\cline { 2 - 3 } & Sí & No \\
\hline 1. ${ }^{\text {er }}$ grupo generacional (60 o más años) & 80,0 & 20,0 \\
\hline 2. $^{\circ}$ grupo generacional (45 a 59 años) & 76,0 & 24,0 \\
\hline 3. $^{\text {er }}$ grupo generacional (25 a 45 años) & 83,0 & 17,0 \\
\hline $4^{\circ}{ }^{\circ}$ grupo generacional (15 a 24 años) & 88,0 & 12,0 \\
\hline
\end{tabular}

Fuente: Investigación Análisis intergeneracional de la vivencia religiosa del poblador de Ayaviri.

Elaboración propia.

En la actualidad, a partir de la influencia de los medios de comunicación, la convivencia aparece como la mejor opción —la más fácil- porque implica menos compromiso que el matrimonio; sin embargo, los pobladores perciben, en su mayoría, un conjunto de diferencias entre ambas situaciones, percepción que se presenta en mayor medida en los jóvenes de la última generación (88\%). Ello se debería a dos factores fundamentales: el primero es el papel que la pastoral actual tiene en la zona, puesto que, al tener una visión más conservadora, permite introducir la importancia de los sacramentos, entre ellos, el matrimonio. Segundo, el hecho de que los jóvenes — hijos y nietos de las personas pertenecientes a las tres primeras generaciones - han visto en sus padres y abuelos las implicancias que ha tenido la convivencia o el matrimonio en sus vidas. 
En el caso de la primera generación (personas que tienen de 60 años a más), entre los discursos vertidos sobre la diferencia entre matrimonio y convivencia, destacan los siguientes:

- La formalidad y la bendición recibida de Dios:

En la convivencia: «A mi parecer, no creo que viven ya formada; a veces separan, a veces no. También peor es, pe. Esa era la diferencia, pero cuando uno está matrimonio, bueno, ya vive, pe, formalmente; más seriedad. Cuando no están casados, pue, claro, no están la ley, la religión».

«Matrimonio es, pe..., uno recibe el sacramento, la bendición del Señor. Es hasta la muerte».

- Seguridad y duración:

«El matrimonio es más seguro; en cambio, en la convivencia, hay esa... un poquito de desconfianza. Qué será. En cambio, habiendo de por medio un matrimonio, hay más seguridad».

- La formalidad y seguridad se extiende a los hijos:

«El matrimonio es una cosa formal. También hay que ver la situación de los hijos. Primero Dios y luego nosotros. La convivencia cualquier rato se pueden separar, se vuelven a unir, no hay formalidad. Los hijos mismos sufren en ese aspecto; en cambio, cuando uno es casado, se crían bien los hijos, el cariño fraternal. 
Ahora, unas mamás los dejan a los hijos, se divorcian; los hijos se trauman».

«Cuando es convivencia, no hay respeto. El matrimonio salva a los hijos».

- Buena imagen social:

«El matrimonio es mejor. Vivimos bien. La gente nos respeta. Cuando no estamos casados, la gente no nos respeta nadie».

«La diferencia es en que el casado lleva una vida respetable, una vida que también respeta al prójimo y a Dios, la Iglesia».

Como se aprecia, los discursos vertidos por los pobladores obedecen a una pastoral tradicional-conservadora que promueve las uniones matrimoniales. Al revisar el estudio intergeneracional sobre la religión en Ayaviri, encontramos que solo en esta pastoral se habla del matrimonio: «Podemos y debemos insistir en que los padres regularicen su situación [se casen] para que presenten a sus hijos al bautismo». ${ }^{12}$ Además, plantean que en la misa de los alferados, no se admita a los convivientes, y piden que estos regularicen su situación. Asimismo, al hablar de los catequistas, se busca que sean casados y, en el caso de que sean convivientes, se les otorga un plazo de tres meses para que «normalicen la situación matrimonial».

12. Conclusiones de las jornadas espirituales del primero de agosto de 1967, folio 207. 
En las siguientes pastorales presentes en la zona y que influyen directamente en la segunda y tercera generación (indigenista-culturalista y político-liberacionista), no se encuentra información alguna sobre el matrimonio, lo que se materializa en los discursos vertidos por los pobladores sobre la diferencia que encuentran en la convivencia, pues la ven como un simple incumplimiento de lo que la Iglesia dice. A consecuencia de ello, aparecen otros aspectos nuevos, como la legalidad de uno frente al otro (matrimonio civil) y la facilidad de la convivencia para separarse ante cualquier conflicto, o el tema económico. La percepción de seguridad, duración y unión ante Dios siguen presentes, pero en una minoría:

- El matrimonio hoy en día ya no se realiza, porque lo natural es convivir:

«El matrimonio es algo formal, ¿no?, que conllevan los esposos ya casados. Ahora, la convivencia es lo natural hoy en día, la costumbre nos aliena. Eso es algo natural, parece, ¿no? Porque según la Biblia dice de que esa convivencia de antes de ser casados antes no se permitía eso. Según la Biblia de que siempre antes de convivir, antes de juntarse dos personas, deben casarse y normal eso era, ¿no? Pero hoy en día es algo natural que las parejas lo mantienen».

- La convivencia es no cumplir lo que Iglesia manda:

«Matrimonio ya... de Dios, con el Dios; convivencia cuando ya no has cumplido con esa misión». 
«Bueno, en el matrimonio me parece que es ya, ¿ah?... ¿Cómo puedo decir? De que la Iglesia, pues, ¿no? obliga de que haiga matrimonio, pero convivir con matrimonio me parece para mí si hay una comprensión igual, ¿no?».

«Solo hay diferencia de que el matrimonio el padre te... te hace casar frente al altar a nombre de Dios; mientras, en cambio, la convivencia estás, ¿cómo te puedo decir?, estás... estás sobre la ley de Dios».

- El matrimonio implica una responsabilidad, mientras que la convivencia, simple libertad:

«El matrimonio ya es una responsabilidad, es bastante responsabilidad y es asumir y compartir. En cambio, cuando uno está conviviendo, es así una vida como medio informal [...]. No es, pues, no es formal. En cambio, tú cuando te casas, adquieres unos votos y tienes que respetar lo que has asumido».

«En el matrimonio hay más responsabilidad. En el caso de una convivencia, dependiendo de las personas, hay personas que lo toman en serio, pue, la familia, y hay personas que no tanto [...]. Llevan una vida no tan seria».

«Cuando uno está matrimonio, creo que tiene que ser más responsable en su hogar y con los hijos más por los hijos; pero, cuando convives, a veces nuestra pareja no lo toma en serio; cualquier rato nos puede dejar, algo así». 
- En el matrimonio no hay facilidad para separarse porque es para toda la vida, en la convivencia sí:

En el matrimonio, «ambos están seguros [...] siempre y cuando piensen lo mismo también, y que ambos estén unidos para toda la vida, pero en la convivencia no es así. Por ejemplo, yo no estoy casada, pero no tengo la obligación de permanecer toda la vida a su lado. Si es que, digamos, que si yo apoyo un maltrato físico, psicológico, ¿por qué tengo que estar aguantando? Total, no estoy casada. Si estuviera casada, tendría que aguantar toda la vida. Yo pienso así es la diferencia. Algunas veces también he conversado con algunas amigas, ¿para qué casarse?... Para aguantarle sus cosas. Siempre hay, a veces, esos problemitas de maltratos físicos, psicológicos. No hay pareja tranquila, o habrá tal vez algunas personas».

«En el matrimonio ya se firma un papel, pero en la convivencia no, pue, o sea, convivimos nomás. Si algún día nos separamos, chau decimos, pero el matrimonio ya no se puede divorciarse, me imagino, ¿no? Para divorciarse, hay que ir a Roma, al obispo, presentar un papel para divorciarse, creo... ¿Es así?».

- El matrimonio como sinónimo de legalidad civil, que genera seguridad a los hijos:

«El matrimonio es un compromiso legal bajo documento y la convivencia no es un trato oficial, puede tener o no la autorización de los padres» 
«La primera [el matrimonio] sería la cuestión legal. Los hijos deben venir a un lugar constituido legalmente. El hijo debe tener dos padres y tener dos apellidos, y la convivencia es el origen de muchas cosas: el abandono familiar, no se sienten ligados, se van y puede haber tenido hijos, y quedan desamparados».

- El matrimonio implica tener mejores ingresos:

«Será, pe, mejor el matrimonio. Una sola persona se mata trabajando sola. Cuando son dos, los dos tienen que partir en el hogar, ¿no?».

- Unión ante Dios, seguridad, duración, respeto e imagen social:

«El matrimonio: estamos bendecidos por Dios, por nuestro Señor y en la convivencia no. Estamos, todo lo contrario, con una falta hacia el Señor».

«Nos prepara espiritualmente, tenemos más tarea, con los hijos, con la familia, con la sociedad misma». «La diferencia es que la persona casada tiene que portarse bien, pues tienen que ser personas dignas; el conviviente, más bien, en cualquier rato se separa, fácil te separas».

En el caso de los más jóvenes (la cuarta generación), las opiniones reflejan en parte la tendencia vista en las dos generaciones anteriores, que hacen referencia a la facilidad de separarse en la 
convivencia, frente a la responsabilidad que genera el vínculo matrimonial. Y al ser hijos de pobladores que muchas veces han convivido, expresan su sentir y su sufrimiento como hijos; por ello, y en vinculación con el quehacer de la nueva pastoral, resurgen los discursos de la primera generación, de reconocer en el matrimonio la bendición de Dios en la Iglesia. Asimismo, vinculan el sacramento con un reconocimiento social de la familia formada:

- La convivencia permite una separación más pronta cuando la pareja no se entiende y, por lo tanto, menos responsabilidades:

«Cuando están casadas, ya asumen una responsabilidad, y cuando conviven así [...], cualquier rato se pueden ir, se pueden separar; a veces no se entienden esas parejas».

«Cuando estás casado, es tener la vida seguro, vivir juntos, seguro; si no estás casado, cualquier rato puede varian».

«El matrimonio es que, digamos, cuando se casan, ya digo “¿Prometen amarse y respetarse?”, todo eso, pero para lo convivio, no se necesita nada».

- Los hijos somos los que más sufrimos:

«Algunos, o sea, conviven, pero no están casados. Ya vienen los hijos. Ese hogar no está feliz [...] porque a veces antes de casarse tienen hijos; entonces, a veces, los hijos somos los que más sufrimos [...] porque 
conviven sin casarse. A veces falta dinero, alimentos, muchas cosas, entonces [...], eso las parejas no han planeado bien, no han planificado, no planificaron bien [...]. Si yo me caso con una persona, tengo que pensar bien, o sea, esa persona va a estar a mi lado siempre toda mi vida, ¿no es cierto? Entonces, para eso, necesito conocerlo todo, eso sí, y por eso hay que tomar una buena decisión; no es... no es juego». «Yo creo que la convivencia no va bien... Como cualquier rato se separan, lo botan, no reconocen hijos; siempre hay más pelea en esa parte».

- El matrimonio como unión bendecida por Dios y ante la sociedad:

«Matrimonio es la unión ante todo público, pues, sobre la Iglesia bendecida por Dios escuchada por testigos [...]. En la Iglesia es la que más vale, está consagrada por Dios».

«Matrimonio es algo que lo une Dios, lo une Dios y, además, permanece en los registros civiles de matrimonio como personas unidas».

«Porque en la convivencia no hay, o sea, no es cumplido un sacramento: el matrimonio ya es diferente pienso, porque, o sea, ya... o sea pienso que Dios y los demás ya saben que tú ya estás casada con una persona».

Por otro lado, si revisamos las estadísticas poblacionales del estado civil, vemos que la convivencia y el matrimonio aparecen 
como dos opciones por seguir, y la convivencia ha venido cobrando fuerza como la opción más fácil, o quizá práctica, para iniciar una relación, tal y como se demuestra en las siguientes estadísticas.

\section{Cuadro 7}

Estado CIVIL SEGÚN GENERACIÓN DE PERTENENCIA (\%)

\begin{tabular}{|l|c|c|c|c|c|}
\hline Generación & Soltero(a) & Casado(a) & Conviviente & Viudo(a) & Divorciado(a) \\
\hline $\begin{array}{l}1 .^{\text {er }} \text { grupo generacional } \\
(60 \text { o más años })\end{array}$ & 19,1 & 51,1 & 10,6 & 14,9 & 4,3 \\
\hline $\begin{array}{l}2^{\circ} \text { grupo generacional } \\
\text { (46 a } 59 \text { años) }\end{array}$ & 13,8 & 44,8 & 37,9 & 3,4 & 0 \\
\hline $\begin{array}{l}3 \cdot^{\text {er }} \text { grupo generacional } \\
(25 \text { a } 45 \text { años })\end{array}$ & 38,3 & 19,8 & 39,5 & 1,2 & 1,2 \\
\hline $\begin{array}{l}4^{\circ} \text { grupo generacional } \\
(15 \text { a } 24 \text { años })\end{array}$ & 94,2 & 0 & 3,8 & 0 & 0 \\
\hline
\end{tabular}

* Para tener un mayor acercamiento a la situación del estado civil y saber qué cantidad de la población opta por el matrimonio, la convivencia o la soltería, podemos considerar que aquellas personas que están viudas y divorciadas pasaron previamente por el matrimonio; con ello, las cifras se incrementarían notoriamente.

Fuente: Investigación Análisis intergeneracional de la vivencia religiosa del poblador de Ayaviri.

Elaboración propia.

Pero la comparación estadística de las personas que refieren estar casadas con aquellas que refieren convivir no agota el fenómeno del crecimiento de los porcentajes de convivencia en la población, pues encontramos que del total de personas casadas, las tres cuartas partes convivieron previamente. Las diferencias generacionales se detallan en el siguiente cuadro: 


\section{Cuadro 8}

DistribuCión POBLACIONAL DE LAS PERSONAS CASADAS, SEGÚN CONVIVENCIA PREVIA AL MATRIMONIO Y GENERACIÓN DE PERTENENCIA

\begin{tabular}{|l|c|c|}
\hline \multirow{2}{*}{ Generación } & \multicolumn{2}{|c|}{ Personas casadas que (\%) } \\
\cline { 2 - 3 } & $\begin{array}{c}\text { Convivieron antes } \\
\text { del matrimonio }\end{array}$ & $\begin{array}{c}\text { No convivieron } \\
\text { antes del matrimonio }\end{array}$ \\
\hline $1 .^{\text {er }}$ grupo generacional (60 o más años) & 68,4 & 31,6 \\
\hline $2^{\circ}$ grupo generacional (45 a 59 años) & 74,1 & 25,9 \\
\hline 3. $^{\text {er }}$ grupo generacional (25 a 45 años) & 83,7 & 16,3 \\
\hline $4^{\circ}{ }^{\circ}$ grupo generacional (15 a 24 años) & 50,0 & 50,0 \\
\hline
\end{tabular}

Fuente: Investigación Análisis intergeneracional de la vivencia religiosa del poblador de Ayaviri.

Elaboración propia.

Entonces, los porcentajes de convivencia se incrementarían de la siguiente manera:

\section{Cuadro 9}

DistribuCIÓN POBLACIONAL SEGÚN GENERACIÓN Y NIVELES DE CONVIVENCIA (\%)

\begin{tabular}{|c|c|c|c|c|}
\hline Generación & Soltero(a) & Casado(a) & $\begin{array}{l}\text { Conviviente (que } \\
\text { actualmente conviven } \\
\text { o que convivieron } \\
\text { antes del matrimonio) }\end{array}$ & $\begin{array}{c}\text { Otros } \\
\text { (viudo o } \\
\text { divorciado) }\end{array}$ \\
\hline $\begin{array}{l}\text { 1. }{ }^{\text {er }} \text { grupo generacional } \\
\text { ( } 60 \text { o más años) }\end{array}$ & 21,0 & 16,0 & 44,0 & 19,0 \\
\hline $\begin{array}{l}\text { 2. }{ }^{\circ} \text { grupo generacional } \\
\text { (46 a } 59 \text { años) }\end{array}$ & 19,0 & 13,0 & 66,0 & 2,0 \\
\hline $\begin{array}{l}3^{\text {er }} \text { grupo generacional } \\
\text { ( } 25 \text { a } 45 \text { años) }\end{array}$ & 41,0 & 3,0 & 54,0 & 2,0 \\
\hline $\begin{array}{l}\text { 4. }^{\circ} \text { grupo generacional } \\
\text { (15 a } 24 \text { años) }\end{array}$ & 95,0 & 0 & 5,0 & 0 \\
\hline
\end{tabular}

Fuente: Investigación Análisis intergeneracional de la vivencia religiosa del poblador de Ayaviri.

Elaboración propia. 
Un tema importante al momento de convivir o casarse es el de las motivaciones que se encuentran tras esa decisión. En el caso de la zona de estudio, encontramos que el embarazo previo a la unión («Tuve mi hija señorita, yo, a los 17») y la presión de los padres («Es que mi mamá, papá, por ellos más que todo, tal vez si no hubiera tenido pareja hubiera continuado mi estudio»), se encuentran por encima de una decisión personal («Por amor, por cariño, pensando que a mi edad ya era adecuado»). En el caso de aquellos que convivieron, la decisión de algunos se da porque no contaron con los primeros sacramentos («Porque uno se enamora, porque, realmente, como sé que no estoy bautizada, nada, creo que todas esas cosas en la religión te piden»).

Por otro lado, nos parece importante analizar el nivel educativo de los pobladores según el estado civil que tienen, a fin de conocer si existe algún grado de relación entre ambos: 
Cuadro 10

Grado de INSTRUCCIÓN DE LOS POBLAdORES SEGÚN EDAD y ESTADO CIVIL DE LOS POBLADORES DE AYAVIRI

\begin{tabular}{|c|c|c|c|c|c|c|c|c|c|c|c|}
\hline \multirow{2}{*}{$\begin{array}{c}\text { Estado } \\
\text { civil }\end{array}$} & \multirow{2}{*}{$\begin{array}{l}\text { Edad } \\
\text { (años) }\end{array}$} & \multicolumn{10}{|c|}{ Grado de instrucción (\%) } \\
\hline & & \begin{tabular}{|c|} 
Sin \\
instrucción
\end{tabular} & $\begin{array}{c}\text { Primaria } \\
\text { incompleta }\end{array}$ & $\begin{array}{l}\text { Primaria } \\
\text { completa }\end{array}$ & $\mid \begin{array}{l}\text { Secundaria } \\
\text { incompleta }\end{array}$ & $\begin{array}{l}\text { Secundaria } \\
\text { completa }\end{array}$ & $\begin{array}{c}\text { Técnico } \\
\text { superior } \\
\text { incomplete }\end{array}$ & \begin{tabular}{|c|} 
Técnico \\
superior \\
completal
\end{tabular} & \begin{tabular}{|c|} 
Superior \\
universitaria \\
incompleta
\end{tabular} & \begin{tabular}{|c|} 
Superior \\
universitaria \\
completa
\end{tabular} & Total \\
\hline \multirow{4}{*}{$\begin{array}{l}\text { Soltero } \\
\text { (a) }\end{array}$} & 15 a 24 & & & 8,3 & 33,3 & 37,5 & 14,6 & 4,2 & 2,1 & & 100 \\
\hline & 25 a 45 & & & 3,2 & & 22,6 & 12,9 & 22,6 & 16,1 & 22,6 & 100 \\
\hline & 45 a 59 & & 25,0 & & 25,0 & 25,0 & & & & 25,0 & 100 \\
\hline & 60 o más & 11,1 & 33,3 & 22,2 & & & & & 11,1 & 22,2 & 100 \\
\hline \multirow{3}{*}{$\begin{array}{l}\text { Casado } \\
\text { (a) }\end{array}$} & 25 a 45 & & 6,3 & 6,3 & 6,3 & 12,5 & & 25,0 & & 43,8 & 100 \\
\hline & 45 a 59 & & 7,7 & 15,4 & 30,8 & & & 7,7 & & 38,5 & 100 \\
\hline & 60 o más & 9,1 & 31,8 & 22,7 & 9,1 & 13,6 & & & 4,5 & 9,1 & 100 \\
\hline \multirow{4}{*}{$\begin{array}{c}\text { Con- } \\
\text { viviente }\end{array}$} & 15 a 24 & & & 50,0 & 50,0 & & & & & & 100 \\
\hline & 25 a 45 & 3,2 & 9,7 & 6,5 & 12,9 & 32,3 & 6,5 & 16,1 & 3,2 & 9,7 & 100 \\
\hline & 45 a 59 & & 18,2 & & 27,3 & 36,4 & & 9,1 & & 9,1 & 100 \\
\hline & 60 o más & 60,0 & & & 20,0 & & & & & 20,0 & 100 \\
\hline \multirow{3}{*}{$\begin{array}{l}\text { Viudo } \\
\text { (a) }\end{array}$} & 25 a 45 & & & & & & & 100,0 & & & 100 \\
\hline & 45 a 59 & & & & 100,0 & & & & & & 100 \\
\hline & 60 o más & 14,3 & 28,6 & 14,3 & 14,3 & 14,3 & & & & 14,3 & 100 \\
\hline \multirow{3}{*}{$\begin{array}{l}\text { Divorciado } \\
\text { (a) }\end{array}$} & 15 a 24 & & & & & & & & & 100,0 & 100 \\
\hline & 25 a 45 & & & & & & & & & 100,0 & 100 \\
\hline & 60 o más & & & 50,0 & 50,0 & & & & & & 100 \\
\hline
\end{tabular}

Fuente: Investigación Análisis intergeneracional de la vivencia religiosa del poblador de Ayaviri.

Elaboración propia.

Como se desprende del cuadro anterior, las personas casadas reportan mayores niveles educativos que las convivientes; entre los convivientes, los niveles educativos se concentran en los secundarios (completos o incompletos). La causa de ello estaría en la mayor estabilidad que un matrimonio brinda desde su constitución, y que permite a sus miembros desplegarse en varios aspectos, entre ellos, el profesional. 


\section{El SERVINÁCUY}

$\mathrm{Al}$ analizar el matrimonio y la convivencia en la zona andina, no podemos dejar de hablar del servinácuy, palabra proveniente del quechua que significa 'mutuos servicios' y que se realiza «antes de celebrar el rito matrimonial religioso y civil» (Marzal 1977: 153). En este sentido, el servinácuy, puede ser entendido como una convivencia previa al matrimonio y eso podría explicar los altos porcentajes de personas que convivieron antes de casarse. Por ello, se indagó sobre la relación que los pobladores establecen entre convivencia y servinácuy, ${ }^{13}$ y se encontraron los siguientes resultados:

\section{Cuadro 11}

Percepción de los Pobladores Sobre LA Similitud

ENTRE EL SERVINÁCUY Y LA CONVIVENCIA

\begin{tabular}{|l|c|c|}
\multirow{2}{*}{ Edad } & \multicolumn{2}{|c|}{ Encuentra similitud (\%) } \\
\cline { 2 - 3 } & Sí & No \\
\hline $1 .^{\text {er }}$ grupo generacional (60 o más años) & 87,0 & 13,0 \\
\hline $2 .^{\circ}$ grupo generacional (45 a 59 años) & 78,0 & 22,0 \\
\hline $3 .^{\text {er }}$ grupo generacional (25 a 45 años) & 71,0 & 29,0 \\
\hline $4 .^{\circ}$ grupo generacional (15 a 24 años) & 32,0 & 68,0 \\
\hline
\end{tabular}

Fuente: Investigación Análisis intergeneracional de la vivencia religiosa del poblador de Ayaviri.

Elaboración propia.

13. En el estudio, no se profundizó en el tema de la significación del servinácuy. Solo se preguntó sobre este punto, al abordar el tema de la convivencia, para ver si los encuestados encontraban similitud entre ambos estados. 
Como se desprende del cuadro, los más adultos encuentran una similitud entre el servinácuy y la convivencia, al significar ambas una unión previa al matrimonio: «La convivencia es una manera de comprenderse una situación prematrimoniah», «es solo convivir, es igual que el servinacuy», «la convivencia es cuando no son casados todavía». Con el pasar de los años, la convivencia ha pasado de ser un paso previo a constituirse en una opción. «Bueno, en mi caso... la convivencia hay que comprendernos con nuestra pareja y seguir adelante sin ser casados», lo que se expresa en una ligera disminución del reconocimiento de las similitudes en la segunda y tercera generación, y se acrecienta de manera fuerte en la última generación, en la que solo $32 \%$ creen que existe una similitud. Entre aquellos que consideran que no existe similitud (68\%), las razones se sustentan principalmente en el desconocimiento del servinácuy, precisamente porque la convivencia ya no es concebida como el paso previo al matrimonio, sino como una alternativa a este. 


\section{REFERENCIAS}

Estenós, Alejandro y Manuel Ugarte

2009 «La Iglesia católica en el altiplano: cinco décadas de trabajo pastoral en la prelatura de Ayaviri (1959-2009)». Allpanchis 73-74, pp. 15-67.

GeErTz, Clifford

1965 «eligion as a Cultural System». En W. Lessa y E. Vogt (eds.). Reader in comparative religion. Nueva York: Harper and Row, pp. 204-216.

JuDD, Esteban

1989 «La inculturación en el contexto andino». Boletin IDEA, serie, 2, 31, abril, pp. 51-87.

Marzal, Manuel

2002 Tierra encantada. Tratado de Antropología religiosa en América Latina. Lima: Trotta-Fondo Editorial de la Pontificia Universidad Católica del Perú.

1988 La transformación religiosa peruana. Lima. Fondo Editorial de la Pontificia Universidad Católica del Perú.

1977 Estudio sobre religión campesina. Lima: Fondo Editorial de la Pontificia Universidad Católica del Perú.

Prelatura de Ayaviri

2012 Información estadística de la Prelatura de Ayaviri, elaborada sobre la base de los Anuarios Pontificios 1967-2008. Disponible en <www.prelaturaayaviri.org>. Recuperado el 10 de enero del 2012. 